\title{
Diffuse large B-cell lymphoma: new targets and novel therapies
}

\author{
Bruce D. Cheson (1)', Grzegorz Nowakowski (10) ${ }^{2}$ and Gilles Salles ${ }^{3}$
}

\begin{abstract}
Newer, more effective and non-cytotoxic therapies are an unmet need for patients with diffuse large B-cell lymphoma $(\mathrm{DLBCL})$ and other B-cell malignancies. Recently approved agents include polatuzumab with bendamustine and rituximab, selinexor, and tafasitamab plus lenalidomide. Three CAR-T cell products are currently approved by the FDA, with others in clinical trials. Additional agents in development include bispecific antibodies and antibody drug conjugates. Combinations of targeted therapies should lead to further improvement in the outcome of patients with B-cell malignancies.
\end{abstract}

\section{Introduction}

The non-Hodgkin lymphomas (NHL) are a diverse group of malignancies about $80 \%$ of which are of B-cell origin (B-NHL). B-NHL vary in their presentation, clinical features, prognosis, and response to treatment. The most common histologic subtype is diffuse large B-cell lymphoma (DLBCL) accounting for about a third of cases in the United States, followed by follicular lymphoma which represents about a quarter ${ }^{1}$. The other histologies are much less common. About $60 \%$ of DLBCL are cured with regimens such as rituximab, cyclophosphamide, Adriamycin, vincristine, and prednisone (R-CHOP $)^{2}$. However, most patients who relapse following or are refractory to initial therapy succumb to their disease ${ }^{3}$. New drug development over the past decade has appropriately ignored cytotoxic chemotherapy drugs and focused on agents that target the cell surface, internal pathways, and the microenvironment. The chimeric anti-CD20 monoclonal rituximab revolutionized the therapy of B-NHL, prolonging survival in most subtypes. However, resistance eventually develops and other strategies directed at other targets are needed.

Recently, several innovative treatments have been approved by the FDA including the anti-CD79b antibody

Correspondence: Bruce D. Cheson (bdcheson@gmail.com)

'Lymphoma Research Foundation, New York, NY, USA

${ }^{2}$ Mayo Clinic Foundation, Rochester, MN, USA

Full list of author information is available at the end of the article drug conjugate polatuzumab vedotin (Pola) with bendamustine and rituximab (Pola-BR $)^{4}$; the oral nuclear transport (XPO1) inhibitor selinexor ${ }^{5}$; and, most recently the combination of the anti-CD19 monoclonal antibody tafasitamab with the immunomodulatory agent lenalidomide $^{6}$. In addition are the first two CART-cell products axicabtagene ciloleucel $^{7}$ and tisagenlecleucel ${ }^{8}$. Other drugs in development that target CD20 include the bispecific T-cell engagers (e.g., mosunetuzumab ${ }^{9}$, glofita$\mathrm{mab}^{10}$, epcoritamab ${ }^{11}$, and odronextamab ${ }^{12}$ ). Each of these has exhibited promising early data, including in patients who have progressed following CAR-T therapy ${ }^{12}$.

Polatuzumab vedotin is an antibody-drug conjugate that targets $\mathrm{CD} 79 \mathrm{~b}$ and delivers the microtubule inhibitor monomethyl auristatin E (MMAE). Polatuzumab vedotin was studied in a phase I, dose escalation trial in 95 patients with NHL or CLL ${ }^{13}$. The primary endpoints were to determine the safety and tolerability, the maximum tolerated dose (MTD), and the recommended phase II dose. In the lymphoma patients, the MTD was $2.4 \mathrm{mg} / \mathrm{kg}$ as a single agent and in combination with rituximab. Grade 3-4 adverse events were reported in $58 \%$ of patients, most commonly neutropenia (40\%), with peripheral sensory neuropathy in $9 \%$. Responses were reported in $54.8 \%$ of the NHL patients. The drug was too toxic and had limited activity to be further pursued in CLL. Pola-BR received accelerated approval for R/R DLBCL after two prior regimens (one prior in the European Union) on the basis of a

(c) (i) Open Access This article is licensed under a Creative Commons Attribution 4.0 International License, which permits use, sharing, adaptation, distribution and reproduction (c) in any medium or format, as long as you give appropriate credit to the original author(s) and the source, provide a link to the Creative Commons license, and indicate if changes were made. The images or other third party material in this article are included in the article's Creative Commons license, unless indicated otherwise in a credit line to the material. If material is not included in the article's Creative Commons license and your intended use is not permitted by statutory regulation or exceeds the permitted use, you will need to obtain permission directly from the copyright holder. To view a copy of this license, visit http://creativecommons.org/licenses/by/4.0/. 
Table 1 Tafasitamab monotherapy vs L-mind regimen in NHL.

\begin{tabular}{|c|c|c|c|c|c|c|}
\hline & $\begin{array}{l}\text { Number of } \\
\text { patients }\end{array}$ & $\begin{array}{l}\text { Overall } \\
\text { response rate }\end{array}$ & $\begin{array}{l}\text { Complete } \\
\text { response rate }\end{array}$ & $\begin{array}{l}\text { Median PFS } \\
\text { (months) }\end{array}$ & $\begin{array}{l}\text { Median DOR } \\
\text { (months) }\end{array}$ & Reference \\
\hline \multirow[t]{2}{*}{ Tafasitamab single agent } & 35 (DLBCL) & $26 \%$ & $6 \%$ & 2.7 & 20.1 & Jurczak 22 \\
\hline & $34(\mathrm{FL})$ & $29 \%$ & $9 \%$ & 8.8 & Not reached & \\
\hline $\begin{array}{l}\text { Lenalidomide } \\
\text { single agent }\end{array}$ & $76(\mathrm{FL})$ & $34.2 \%$ & $13.2 \%$ & 4.0 & 6.6 & Nowakowski ${ }^{25}$ \\
\hline $\begin{array}{l}\text { Tafasitamab and } \\
\text { lenalidomide }\end{array}$ & 81 (DLBCL) & $60 \%$ & $42.5 \%$ & 12.1 & 21.7 & Salles ${ }^{6}$ \\
\hline
\end{tabular}

$D L B C L$ diffuse large B-cell lymphoma, $F L$ follicular lymphoma, $P F S$ progression-free survival, $D O R$ duration of response.

randomized trial comparing Pola-BR with BR, with 40 patients per arm in which Pola-BR achieved a higher complete remission $(\mathrm{CR})$ rate $(40.0$ vs $17.5 \%, p<.026)$ and a longer progression-free survival (PFS) $(9.5$ months vs 3.7 months $(p=.001)$ and overall survival (OS) (12.4 vs 4.7 months, $p=.002$ ). (Table 1) However, Pola-BR was associated with more grade 3-4 neutropenia, anemia, and thrombocytopenia as well as grade 1-2 peripheral neuropathy ${ }^{4}$.

Selinexor is an inhibitor of exportin 1 (XPO1), the major nuclear export protein for a number of tumor suppressor genes and proto-oncogenes. Elevated XPO1 expression inactivates tumor suppressor proteins by mislocalization. Selinexor is a specific inhibitor of XPO1, it reactivates tumor suppressor proteins and blocks protooncogene translation, DNA damage repair. The initial phase I trial included 79 patients with NHL, 43 of which had relapse or refractory DLBCL ${ }^{14}$. The most common adverse events included thrombocytopenia in $47 \%$, neutropenia in $32 \%$ and fatigue in $11 \%$, with hyponatremia in $10 \%$. In DLBCL, the ORR was $32 \%$ with CR in $10 \%$ and mDOR of 12.8 months. Activity was also noted in small numbers of patients with follicular lymphoma, CLL, Richter transformation, mantle cell and T-cell lymphomas. The recommended phase 2 dose was $60 \mathrm{mg}$ orally twice weekly. Selinexor received accelerated approval for $\mathrm{R} / \mathrm{R}$ or transformed DLBCL following two prior regimens on the basis of the SADAL single arm trial in patients with de novo or transformed DLBCL not considered eligible for autologous stem cell transplantation (ASCT) or post$\mathrm{ASCT}^{5}$. These 134 patients had a median age of 67 years, median of two prior regimens, with 53\% progressing within a year of their first therapy for DLBCL. This oral agent achieved an ORR of $28 \%$ including $13 \%$ CRs and with a median duration of response of 9.3 months, but was 23 months for the CRs. At the $60 \mathrm{mg}$ twice weekly dose used in this study, and with intensive anti-emetic support, the drug was well tolerated. The most common toxicity was fatigue in $63 \%$, which was grade 3 or 4 in $15 \%$. Other grade 3-4 toxicities were uncommon. In a subsequent analysis including 134 patients, those $<65$ years had an ORR of 36.5 vs $24.4 \%$ for the older patients, CRs 17.3 and $11 \%$, and median duration of response (DOR) of 9.7 and 9.2 months, respectively.

There have been concerns of a potential favorable selection bias in the SADAL trial in that patients could not have had primary refractory disease, and those with a previous CR or partial remission (PR) to their prior line of therapy were required to wait 60 days from that treatment to initiate selinexor, and 98 days for those with refractory disease $^{15}$. The actual time from progression of disease to selinexor therapy was 1.5 months and 3.3 months, respectively. However, patients in the SADAL study were comparable to typical patients given the patient age, amount of prior therapy. Moreover, 30\% had progressed after an autologous stem cell transplant and $72 \%$ were refractory to their immediately prior treatment regimen. In addition, the median time from disease progression from the last prior therapy was 59 days in the selinexor responders compared with 52 days in the non-responders, demonstrating that response did not correlate with time since last therapy.

\section{Targeting CD19}

Another potential target is the CD19 antigen. CD19 is a $95 \mathrm{kd}$, type I, transmembane glycoprotein. Expression of CD19 is specific to B-lymphocytes and follicular dendritic cells on which it is ubiquitous. Expression of CD19 on cells of B-lineage can be through the various stages of differentiation from pre-B cells until plasma cells. CD19 functions as a positive regulator of B-cell receptor (BCR) signaling and is critical for B-cell development, and, in mice the ability to mount an immune response to mitogens, and the production of serum immunoglobulins ${ }^{16}$.

CD19 is present on malignant cells from the majority of patients with NHL, acute lymphoblastic leukemia (ALL) and chronic lymphocytic leukemia (CLL). While CD20 has a higher average density of surface molecules per tumor cell, CD19 expression is more homogenous and is preserved in small CD20-negative tumor subsets and after 


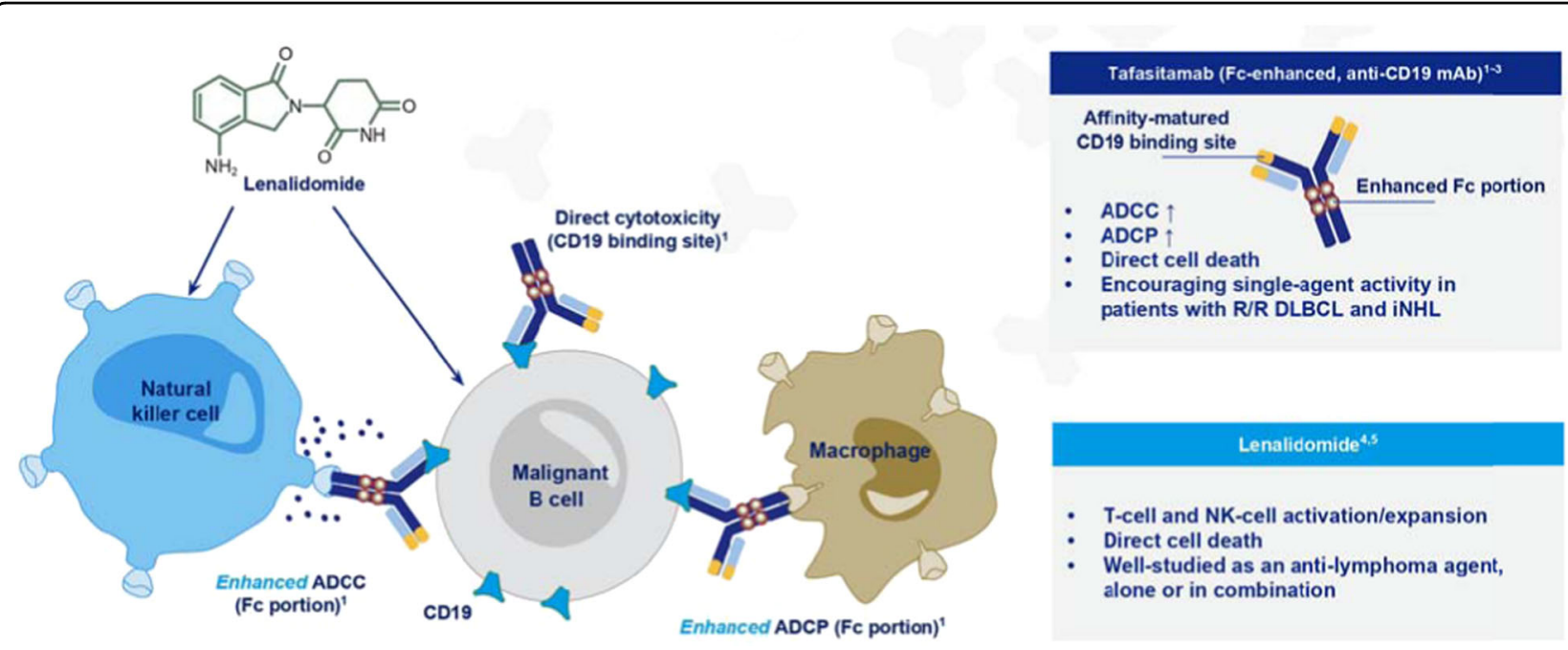

Fig. 1 Mechanism of action of tafasitamab and lenalidomide. Tafasitamab has been Fc enhanced to increase antibody-dependent cellular cytotoxicity (ADCC) which augments interactions with natural killer (NK) cells activated and expanded by lenalidomide, antibody-dependent cellular phagocytosis (ADCP) to enhance interactions with macrophages, and direct cell death.

anti-CD20 targeted therapy. Thus, CD19 serves as an attractive target for lymphoma therapies.

Agents currently in development that target CD19 include tafasitamab, antibody drug conjugates such as loncastuximab tesirine ${ }^{17}$, bispecific T-cell engagers, and CART-cell products including lisocaptagene maraleucel, which was recently FDA approved ${ }^{18}$. Loncastuximab teserine is an antibody-drug conjugate comprised of a humanized anti-CD19 monoclonal antibody conjugated to SG3199, a pyrrolobenzodiazepine dimer toxin. In the phase $\mathrm{I}^{17}, 88$ patients with relapsed or refractory NHL and a median of three prior regimens were treated with loncastuximab teserine at doses escalating from 15-200 $\mu \mathrm{g} /$ $\mathrm{kg}$. The most common treatment emergent adverse events (TEAEs) included hematologic abnormalities, fatigue, liver chemistry elevations, nausea, rash, and dyspnea. At doses of $\geq 150 \mu \mathrm{g} / \mathrm{kg}$, the overall response rate was $59.4 \%$, including $40.6 \%$ CRs. In the subsequent final report including the dose expansion cohort ${ }^{19}$. one hundred and eighty-three patients were included, with the expansion patients treated at either $\geq 120 \mu \mathrm{g} / \mathrm{kg}$ or $\geq 150 \mu \mathrm{g} / \mathrm{kg}$. An MTD was not reached, but $\geq 150 \mu \mathrm{g} / \mathrm{kg}$ was chosen as the RP2 dose. The most common TEAEs included febrile neutropenia, fever, pleural effusion, dyspnea, and sepsis. Thirty-five (19.1\%) patients experienced TEAEs with a fatal outcome during the study, most commonly (20/35) due to progression of underlying NHL. Six were considered treatment related, all of which were infections. Peripheral edema, pericardial or pleural effusions, and ascites were common and problematic, and elevated liver chemistries were common. For the DLBCL cohort, the ORR was $42.3 \%$, with $23.4 \%$ CR; the median DOR was not reached, and the PFS was only 2.8 months.

\section{Tafasitamab}

Tafasitamab is an Fc enhanced, humanized anti-CD19 IgG1/IgG2 monoclonal antibody that was engineered to have increased antibody dependent cellular cytotoxicity (ADCC) and antibody dependent cellular phagocytosis (ADCP) with increased cell killing (Fig. 1). The activity of monoclonal antibodies is dependent on interactions with FcyRII, FcyRIII, and FcyRI receptors on effector cells. Natural killer (NK) cells solely express the FcyRIIIa receptor. The majority of monoclonal antibodies in clinical use, such as rituximab, require an interaction between their Fc domain and FcyRIIIa receptor on NK cells for their potency. Tafasitamab has an Fc variant with a twoamino acid substitution at S239D and I332E which increases its affinity to $F c \gamma R$. The remaining protein sequence of tafasitamab is identical to the IgG1 subclass of monoclonal antibodies in the Fab and hinge regions and identical to the IgG2 subclass of monoclonal antibodies in the $\mathrm{CH} 2$ and $\mathrm{CH} 3$ domains.

Tafasitamab has demonstrated additive or synergistic activity in vitro with a number of chemotherapy agents including bendamustine, fludarabine, rituximab, and ofatumumab.

\section{Phase I study}

In an initial phase I trial, tafasitamab was administered to 27 patients with CLL at doses escalating from $0.3-12 \mathrm{mg} / \mathrm{kg}$, for nine infusions on days $1,4,8,15$, and 22 of cycle 1 and days $1,8,15$, and 22 of cycle 2 . Following the two cycles, patients without progressive disease were allowed to continue tafasitamab every 28 days for four additional infusions ${ }^{20}$. After demonstrating excellent tolerability, an expansion cohort was treated at $12 \mathrm{mg} / \mathrm{kg}$. All 
patients experienced at least one TEAE, 24 of which were thought possibly related to the study drug. Grade 3 or 4 toxicities included neutropenia (11\%) (including a DLT of grade 4 neutropenia), thrombocytopenia (7.4\%), elevated aspartate aminotransferase (3.7\%), and tumor lysis syndrome (3.7\%). Clinical responses occurred in $66.7 \%$ with $29.8 \%$ responding by CT criteria.

Pharmacokinetic studies were performed on 25 patients and showed a two compartment model. The clearance and volume of distribution were similar to other full length monoclonal antibodies with a maximum concentration (Cmax) increasing in a somewhat less than a dose-proportional manner supporting that distribution was limited to the systemic circulation. The clearance and half-life were not dose dependent. There was a trend of accumulation in concentration with each infusion achieving a plateau state by infusion 9 . In the studied range from 3-12 $\mathrm{mg}$, the drug half-life averaged 13.5 days allowing dosing intervals of 1 to 3 weeks.

\section{Phase II studies in CLL}

The phase I data supported the COSMOS study of tafasitamab in patients with CLL and small lymphocytic lymphoma (SLL) - who had progressed following a Bruton tyrosine kinase (BTK) inhibitor ${ }^{21}$. Therapy involved treatment with tafasitamab and either the PI3k inhibitor idelalisib (Cohort A) or venetoclax (Cohort B). Tafasita$\mathrm{mab}(12 \mathrm{mg} / \mathrm{kg})$ was administered in 28 -day cycles. In cycles $1-3$ tafasitamab was infused on day 1 , day 8 , day 15 , and day 22 with an additional loading dose on day 4 of cycle 1 . In subsequent cycles, tafasitamab was administered on days 1 and 15 for cycle 4-6 monthly cycles, and only on day 1 from cycle 7 to 24 or until disease progression or intolerance. Cohort A enrolled 11 patients who received a median of five prior lines of therapy, more than half of which had a complex karyotype or del17p/ TP53. The most common grade 3 or higher TEAEs were neutropenia (46\%), anemia (27\%), thrombocytopenia (27\%), pneumonia (27\%), and elevated transaminases (18\%). Fatal heart failure occurred in one patient. The ORR in Cohort A was 90.9\% with 9.1\% CRs. Two of eight patients who were assessed for minimal residual disease (MRD) status were undetectable in the peripheral blood (PB), and one of three in the bone marrow (BM). Cohort B included 13 patients who had received a median of three prior lines of treatment, more than $90 \%$ of which had a complex karyotype and $69 \%$ unmutated IGHV status. The most common grade 3 or higher TEAEs were neutropenia (46\%), hypophosphatemia (31\%), and infusion related reactions (IRR) (15\%). The ORR was $76.9 \%$ with $23.1 \%$ CRs. Of the seven patients assessed for MRD status, six became undetectable in the $\mathrm{PB}$, and two of four patients in the BM.

\section{Phase II studies in non-hodgkin lymphoma}

Tafasitamab has been studied most extensively in NHL. The first phase II trial involved 92 patients with various histologies of NHL including 35 DLBCL, 34 FL, $12 \mathrm{MCL}$, and 11 other indolent non-Hodgkin lymphoma (iNHL) ${ }^{22}$. Tafasitamab was administered at a dose of $12 \mathrm{mg} / \mathrm{kg}$ on day $1,8,15$, and 22 of cycles 1 and 2, then every 2 weeks onward, and patients who achieved at least a PR were eligible to continue treatment until intolerance or progression of disease. The ORR in relapsed/refractory DLBCL, FL, and other iNHL was 26,29 , and $27 \%$, respectively (Table 1 ). Of six patients with mantle cell lymphoma, there were no responses and this cohort was terminated. The most commonly reported TEAEs were IRR and neutropenia, both occurring in $12 \%$ of patients. Respiratory tract infection and headache were reported in $11 \%$ each. The most common grade 3 or higher adverse event was neutropenia (9\%) which mainly occurred during the first two cycles, with recovery within 1 week (Table 3). The incidence of serious adverse events (SAEs) in the study was 30\%; however, in only four patients (4\%) was there a suspected relationship to tafasitamab. Two SAEs occurred in patients with DLBCL (febrile neutropenia and genital herpes) and two with FL (dyspnea and myelodysplastic syndrome). There were no treatment related deaths.

\section{Combination regimens}

Supported by the modest single agent response rate of tafasitamab in DLBCL, combinations with other agents were developed. To date, the most promising of partners has been the immunomodulatory drug, lenalidomide. The rationale is that the antibody augments cellular immunity against the lymphoma cell target. The Fc enhanced portion of tafasitamab has increased affinity to $\mathrm{Fc} \gamma$ receptors such as FcyRIIIa. These FcyRIIIa receptors, present on immune effector cells such as NK cells, mediate the ADCC response. Lower PB NK cell count has been associated with poorer clinical outcomes in patients with DLBCL supporting an essential role of NK cells in ADCC. In addition, the antibody induces ADCP, augmenting macrophage cell killing. Lenalidomide has been well studied in lymphoma both as a single agent and in combinations. In vitro studies demonstrated that NK-cell mediated ADCC with tafasitamab was further enhanced by lenalidomide.

These observations led to the L-MIND study, a phase II trial of the combination of lenalidomide and tafasitamab (Fig. 2) ${ }^{6}$. Eligibility required age of at least 18 years with relapsed or refractory (but not primary refractory) DLBCL and ECOG performance status of $0-2$. Patients could not be considered eligible for ASCT by their primary physician on the basis of age or the presence of comorbidities. A small subset entered the trial after refusal of ASCT. Tafasitamab was administered at a dose of $12 \mathrm{mg} / \mathrm{kg}$ iv on 


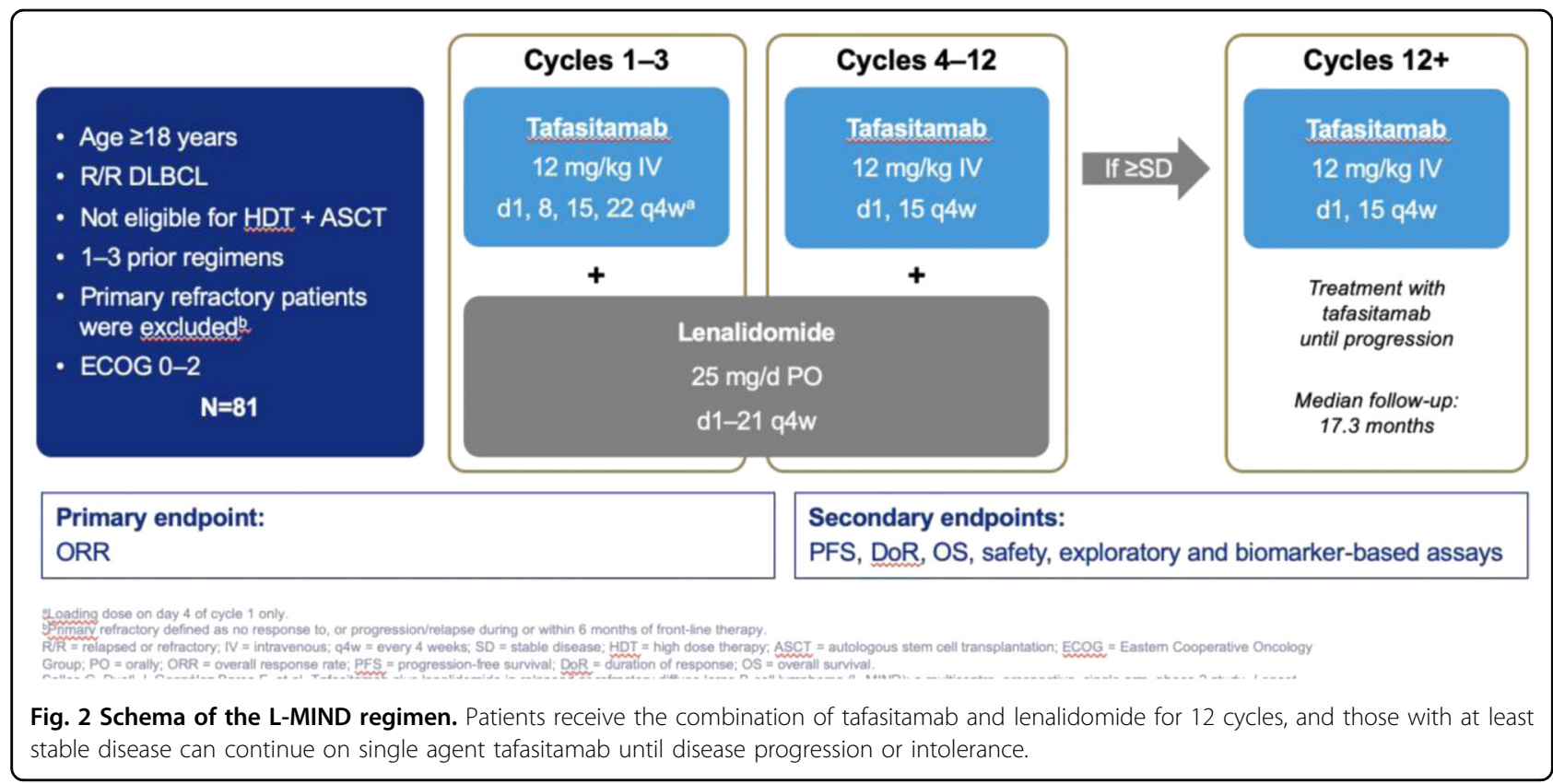

days $1,4,8,15$, and 22 for cycle 1 , days $1,8,15$, and 22 for cycles $2-3$, and days 1 and 15 for subsequent cycles. Lenalidomide was dosed at $25 \mathrm{mg}$ a day, day 1-21 of each 28-day cycle, higher than the $20 \mathrm{mg}$ dose used in the rituximab plus lenalidomide regimen. Patients with stable disease or better after 12 monthly cycles were eligible to continue tafasitamab as a single agent until disease progression or intolerance. The primary endpoint was ORR, with secondary endpoints including PFS, DOR, safety and a number of exploratory biomarker studies.

There were 81 patients accrued to the study. Patient characteristics included a median age of 72 (41-86) years. Fifty-four percent were male and $75 \%$ had stage III or IV disease. IPI $0-2$ were in $49 \%$ and an elevated LDH was noted in 56\%. These patients had limited adverse features. Whereas they had received a median of two prior lines of therapy, $49 \%$ only had one prior and $43 \%$ two prior therapies. Only 15 patients $(18 \%)$ were considered to have primary refractory disease. Such patients were not initially considered eligible for the study; however, the definition of "primary refractory" changed during the conduct of the trial from progression within 3 months to progression within 6 months and the 15 patients had been within the 3-6 month window. In addition, 44\% were considered refractory to their prior line of therapy. Eleven percent underwent a prior stem cell transplant. Cell of origin was germinal center B-cell (GCB) in $46 \%$, non-GCB in $25 \%$, and unknown in $30 \%$. Of note is that CART-cell patients are also a selected population, and the $40 \%$ long-term duration of response is not dissimilar from that reported with either autologous stem cell transplant or L-MIND in transplant ineligible patients.
In updated results, as of November 2019, the ORR was $58.8 \%$ with $41.3 \% \mathrm{CRs}^{23}$. The median PFS was 16.2 months with a median survival of 31.6 months (Fig. 3). With at least 24 months of follow-up, the median DOR was 34.6 months (Fig. 4). Of note was that the median DOR was not reached for the CRs, but was 5.6 months for the PRs, confirming the importance of achieving a CR. At the time of this publication there were 22 patients still on therapy.

This regimen was well-tolerated with the major adverse effects being neutropenia: grade 3 and 4 in 27 and 21\%, respectively, but without infections. Grade 3 thrombocytopenia was reported in $17 \%$. Non-hematologic toxicities included rash (grade 1-2 in 27\%, grade 3 in $9 \%$ ); grade $1-2$ diarrhea in $32 \%$, but grade 3 in only $1 \%$; other toxicities occurring in at least $20 \%$ of patients included asthenia (21\%), cough (21\%), peripheral edema (22\%), fever (20\%), and decreased appetite (20\%); however, grade 3 or worse toxicities were uncommon (Table 2). Treatment related SAE occurred in $18.5 \%$ of patients with $10 \%$ experiencing infections and 5\% with febrile neutropenia. There were four treatment related deaths (sudden death, respiratory failure, cerebrovascular accident, progressive multifocal leukoencephalopathy), none thought to be related to study drugs. The marked reduction in toxicity with the prolonged tafasitamab as a single agent suggests that the toxicity during induction was primarily the result of lenalidomide. Despite the $25 \mathrm{mg}$ starting dose of lenalidomide, $80 \%$ of patients were able to remain on at least $20 \mathrm{mg}$. About half the patients received growth factor support at the discretion of the treating physician in order to maintain dose intensity, which is considered important for the efficacy of the regimen. 

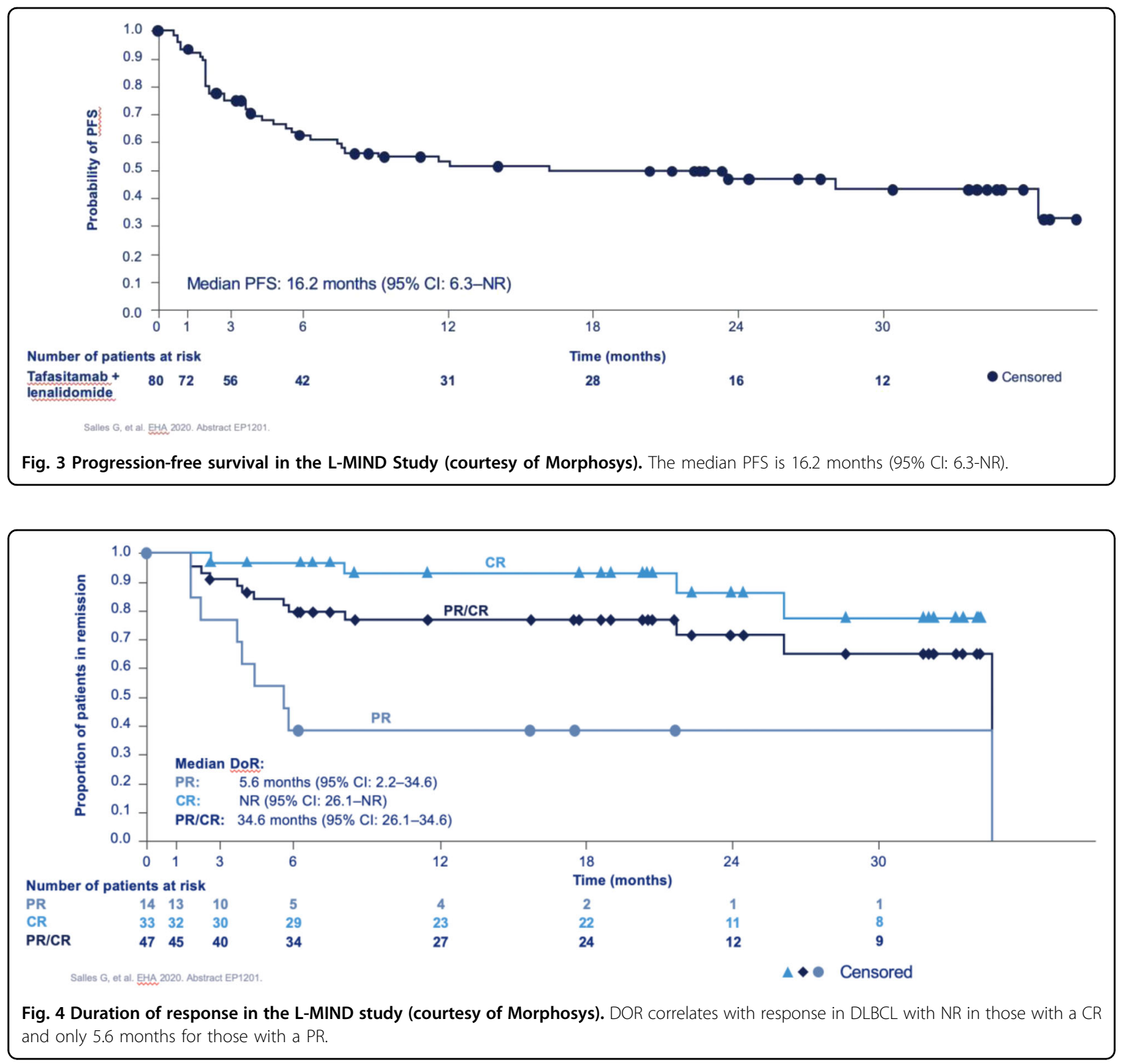

In the L-MIND study, grade 3 and 4 neutropenia were reported in 27 and $21 \%$, respectively; however, febrile neutropenia in only 10 and $2 \%$, respectively. Neutropenia was managed by granulocyte colony-stimulating factor in $44 \%$ of patients, including $81 \%$ of those with grade 3 or 4 neutropenia. The majority of these cases returned to normal neutrophil count within a week.

Management of neutropenia with L-MIND is similar to the published recommendations for ritiuximab plus lenalidamide $\left(R^{2}\right)^{24}$. When neutrophils fall below $1000 / \mu \mathrm{l}$ for at least 7 days or below $1000 / \mu \mathrm{l}$ in the setting of a fever $>38.5^{\circ} \mathrm{C}$, or $<500 / \mu \mathrm{l}$, lenalidomide should be interrupted and blood counts checked weekly. When neutrophils return to $\geq 1000 / \mu \mathrm{l}$, resume at a dose $5 \mathrm{mg} /$ day less than the starting dose. The routine prophylactic use of a growth factor is not recommended; however, such an agent should be considered in the setting of grade 3 or 4 neutropenia lasting $\geq 7$ days or in patients with recurrent neutropenia to maintain the lenalidomide dose.

The efficacy of L-MIND appears to be superior to the $29 \%$ ORR previously reported with tafasitamab monotherapy (Table 1). However, at the request of the FDA, the RE-MIND study was designed as a non-interventional data comparison of patients treated with L-MIND with those treated with single agent lenalidomide who were matched for nine relevant clinical and laboratory features; age, stage, refractoriness to last line of therapy, number of prior therapies, history of prior refractoriness, prior 
Table 2 Adverse events of grade 3 or higher encountered in NHL patients in phase II studies.

\begin{tabular}{|c|c|c|}
\hline Events & $\begin{array}{l}\text { Tafasitamab } \\
\text { single agent }^{\mathrm{a}}\end{array}$ & $\begin{array}{l}\text { Tafasitamab and } \\
\text { lenalidomide }^{b}\end{array}$ \\
\hline Neutropenia & $9 \%$ & $48 \%$ \\
\hline Thrombocytopenia & $4 \%$ & $17 \%$ \\
\hline Anemia & $3 \%$ & $7 \%$ \\
\hline Febrile neutropenia & - & $2 \%$ \\
\hline Dyspnea & $4 \%$ & - \\
\hline Pneumonia & $3 \%$ & $6 \%$ \\
\hline Fatigue & $2 \%$ & $2 \%$ \\
\hline Hypokalemia & $2 \%$ & $1 \%$ \\
\hline Rashes & - & $9 \%$ \\
\hline Hypokalemia & - & $6 \%$ \\
\hline Urinary tract infection & - & $5 \%$ \\
\hline Hypertension & - & $4 \%$ \\
\hline $\begin{array}{l}\text { Pulmonary embolism and } \\
\text { deep vein thrombosis }\end{array}$ & - & $4 \%$ \\
\hline Atrial fibrillation & - & $3 \%$ \\
\hline $\begin{array}{l}\text { Upper respiratory tract } \\
\text { infection }\end{array}$ & - & $2 \%$ \\
\hline Congestive heart failure & - & $2 \%$ \\
\hline Increased transaminases & - & $2 \%$ \\
\hline Renal failure & - & $2 \%$ \\
\hline Back pain & - & $2 \%$ \\
\hline Asthenia & - & $2 \%$ \\
\hline
\end{tabular}

${ }^{a}$ Encountered in two or more patients.

${ }^{b}$ In at least $2 \%$ of the patients.

ASCT, neutropenia, anemia, and elevated $\mathrm{LDH}^{25}$. The primary endpoint was best ORR determined by the investigator. There were 490 patients identified with lenalidomide therapy alone of which 76 matched with 76 of those patients from the L-MIND study. The ORR and CR rates with L-MIND were $67.1 \%$ and $39.5 \%$, respectively, and for lenalidomide monotherapy $34.2 \%$ and $13.2 \%$, respectively $(p<0.0001)$. The PFS was 12.1 months vs 4 months ( $p=.0002$; HR 95\% CI $0.307-0.698)$ and overall survival was also significantly longer at not reached vs 9.4 months for the L-MIND regimen vs lenalidomide monotherapy, respectively $(p=.0026)$ (HR 95\% CI, 0.317-0.785) (Table 1). RE-MIND2 is being planned in which the data with tafasitamab and lenalidomide will be compared in a similar fashion with patients treated with a variety of other options.

Based on these data, L-MIND was granted accelerated approval by the FDA on August 3, 2020.
Whether L-MIND is superior to $\mathrm{R}^{2}$ in DLBCL is unknown as there have been no direct comparisons. Wang et al. reported on 32 patients with relapsed or refractory DLBCL, transformed or grade 3 follicular lymphoma at a median age of 65 years and with a median of three prior lines of therapy ${ }^{26}$. The ORR was $33 \%$ with $22 \%$ CR, a median PFS of 2.8 months and a median OS of 10.2 months. For the DLBCL subset $(n=32)$, the ORR was $28 \%$ including $22 \%$ CRs. Zinzani et al. ${ }^{27}$ reported a series of 23 elderly patients with relapsed or refractory disease. The ORR was $35 \%$ with $30 \% \mathrm{CR}$, a 1-year diseasefree survival rate of $34.8 \%$ and an 18 month OS of $55.1 \%$. Despite differences in patients, the results with L-MIND appear superior to either of these studies.

Other tafasitamab combinations are in development. The B-MIND study is the confirmatory trial for L-MIND and it is an ongoing comparison between bendamustine and rituximab vs bendamustine tafasitamab in relapsed and refractory DLBCL. FIRST-MIND is a phase $1 \mathrm{~b}$ study exploring the relative tolerability of R-CHOP-tafasitamab vs $\mathrm{R}$-CHOP-tafasitabmab+lenalidomide. The more favorable regimen will likely be compared with R-CHOP in a phase III trial in previously untreated DLBCL.

\section{Toxicities of tafasitamab}

Tafasitamab as a single agent and in combination with lenalidomide is generally well tolerated. The most common adverse events are listed in Table 2 which suggests that most of the toxicities in the L-MIND study were related to the lenalidomide.

\section{Tafasitamab administration and dose modifications}

Patient treated with tafasitamab should receive premedication with acetaminophen, histamine $\mathrm{H}_{2}$ receptor antagonists and/or glucocorticoids. Premedication should be administered 30 min to $2 \mathrm{~h}$ prior to starting tafasitamab administration. Subsequent premedication is optional for patients who do not experience an infusion-related reaction during the first three infusions. However, patients who continue to experience infusion-related reactions should receive premedication before each subsequent infusion. The dose of tafasitamab is $12 \mathrm{mg} / \mathrm{kg}$ intravenously days $1,4,8,15$, and 22 of cycle 1 ; days $1,8,15$, and 22 of cycles 2 and 3; days 1 and 15 of cycles 4-12 and for subsequent cycles for patients whose response is at least stable disease until progression of disease or intolerance. The first infusion should be administered at a rate of $70 \mathrm{~mL} / \mathrm{h}$ for the first $30 \mathrm{~min}$, then, the rate can be increased, in the absence of an infusion reaction, so that the infusion is administered within 1.5 to $2.5 \mathrm{~h}$. All subsequent infusions can be delivered within 1.5 to $2 \mathrm{~h}$.

Dose modification recommendations for hematologic toxicity are outlined in Table 3. 
Table 3 Dose modifications for tafasitamab and lenalidomide hematologic toxicities.

\begin{tabular}{ll}
\hline Event & Dose modification \\
\hline Platelet count $\leq 50,000 / \mu \mathrm{l}$ & Hold tafasitamab and lenalidomide and monitor counts weekly until \\
& platelet count is $\geq 50,000 / \mu l$ \\
$\begin{array}{l}\text { Neutrophil count } \leq 1000 / \mu \mathrm{l} \text { for at least } 7 \text { days or febrile neutropenia or } \\
\text { neutrophil count } \leq 500 / \mu \mathrm{l}\end{array}$ & $\begin{array}{l}\text { Hold tafasitamab and lenalidomide and monitor counts weekly until } \\
\text { neutrophil count is } \geq 1000 / \mu l\end{array}$ \\
\hline
\end{tabular}

Table 4 Response and outcomes results for regimens for the treatment of relapsed and refractory DLBCL.

\begin{tabular}{|c|c|c|c|c|c|c|}
\hline Efficacy metric & Selinexor $(n=134)$ & Pola-BR $(n=40)$ & L-MIND $(n=81)$ & R-GemOX $(n=49)$ & R-GDP $(n=52)$ & $\mathrm{R}^{2}(n=32)$ \\
\hline ORR, \% & 29 & 45 & 57.5 & 61 & 63 & 28 \\
\hline$C R, \%$ & 13 & 40 & 40 & 44 & 31 & 22 \\
\hline mDOR (mo) & 9.3 & 12.6 & 34.6 & 10 & $N R$ & 6 \\
\hline mPFS (mo) & 2.6 & 9.5 & 12.1 & 5 & $3 y r-31 \%$ & 2.8 \\
\hline mOS (mo) & 9.0 & 12.4 & 31.6 & 11 & $3 y r-66 \%$ & 10.2 \\
\hline
\end{tabular}

\section{Future directions}

Several active targeted therapies have been approved by the FDA for patients with relapsed or refractory DLBCL, with numerous others in development. The L-MIND regimen is the first therapy approved for second and subsequent lines of therapy patients not considered candidates for ASCT. As noted above, studies are ongoing and planned to determine the role of tafasitamab as part of initial therapy for patients with this histology. Patients with DLBCL are often elderly with comorbidities. Combining L-MIND with mini-CHOP or comparing L-MIND + rituximab with mini-CHOP are questions worthy of study in such a population.

Patients with relapsed or refractory disease have progressed after anti-CD20 therapy. Thus, switching to CD19-direted therapy may improve patient outcome. Incorporating tafasitamab into a pre-ASCT regimen is a question worth study.

Concerns have been expressed that using an anti-CD19 agent may compromise subsequent CART-cell therapy. However, levels of CD19 are maintained following tafasitamab therapy and anecdotes suggest successful cellular therapy in patients previously treated with L-MIND. CART-cell therapy is an extremely promising therapy for DLBCL, MCL, and FL. However, the majority of patients do not benefit and face disease progression. Tafasitamabbased therapy should be tested in this setting, except in those patients whose cells no longer express CD19.

Based on their mechanism of action, the activity of targeted agents as anti-CD47 antibodies, check point inhibitors, or bispecific T-cell engagers, might be augmented by L-MIND.
As CD19 is expressed on virtually all B-cell malignancies, a logical step is to test tafasitamab alone or with lenalidomide in other histologies, notably FL and marginal zone lymphoma (MZL). Since early studies did not suggest activity in MCL, further pursuit is of lower priority. The rituximab/lenalidomide $\left(R^{2}\right)$ regimen has been shown to be effective in the relapsed/refractory and front-line settings. Whether substituting tafasitamab for rituximab or adding the two antibodies are two approaches that should be considered. Combinations of monoclonal antibodies in lymphoid malignancies has been shown to be effective in earlier studies. Such regimens could not only be studied in the relapsed refractory setting, as in the AUGMENT study, but also in the front-line setting. Other combinations with PI3k or BTK inhibitors may be worthy of testing in appropriate histologies. Future directions include combinations with other agents or substitution for rituximab in patients previously treated with the antiCD20 monoclonal antibody.

Tafasitamab may also serve as a backbone for the development of novel strategies for patients with other histologies of NHL.

Having an increasing number of active therapies for DLBCL raises a number of issues. First is how best to sequence them. At present, L-MIND is the only therapy approved for patients failing a single prior systemic regimen. For patients progressing after this regimen and not suitable for CART therapy, the decision is between selinexor and Pola-BR (Table 4). The former has an advantage for ease of administration and a favorable safety profile. Whereas the latter achieves a higher CR rate and longer PFS, the DOR rates were comparable. Moreover, 
an advantage for selinexor is its ease of incorporation into combination strategies, many of which are currently in development.

Thus, with the increasing availability and approval of novel therapies for DLBCL, recommendations for an optimal paradigm is challenging and dynamic. At present, the L-MIND regimen is the only FDA approved secondline therapy, although polatuzumab $+\mathrm{BR}$ has recently received compendium status. CART therapy is currently approved in the third line; however, three ongoing trials are evaluating this therapy in the second line (axicaptagene ciloleucel, tisagenlecleucel, and lisocaptagene maroleucel), and one in front-line, high-risk patients ${ }^{28}$. Thus, patients who are relapsed/refractory to $\mathrm{R}-\mathrm{CHOP}$ are distinguished by their eligibility for stem cell transplantation. For those who are not, L-MIND is the approved option. Therapies for the third line include CAR $\mathrm{T}$ for those who are eligible and who have access to the procedure, or selinexor and poltazumab-BR; the oral administration and safety profile of the former are in its favor; however, the CR rate and PFS support the latter. Should loncastuximab teserine receive approval, its position in the paradigm will be determined by the line of treatment for which is indicated.

How best to move these regimens earlier in the course of the disease where they are likely to be more effective is an important consideration. The POLARIX trial comparing $\mathrm{R}-\mathrm{CHOP}$ with $\mathrm{R}-\mathrm{CHP}+$ polatuzumab has been completed. If the results favor the investigational arm, LMIND would remain as second line followed by selinexor or CART. If the upcoming tafasitamab- $\mathrm{R}^{2} \mathrm{CHOP}$ vs $\mathrm{R}$ CHOP front-line study is positive, it would become a preferred option for initial therapy. If both are positive, treating physicians would have to decide between them on the basis of relative safety and efficacy. Tafasitamab use in later lines of therapy would then be limited to patients who had not already received that agent. For the other patients, Pola-BR in patients naive to those drugs would be a consideration as it has compendium acceptance in second line. Should CAR T be approved in the second line, the selection of therapy among CART T, L-MIND, and Pola-BR would be determined by eligibility criteria, availability, toxicity concerns, financial considerations, and other factors. ADCs, bispecifics, and CART approaches are rapidly moving earlier in the course of the disease. Indeed, mosunetuzumab has been evaluated as the initial line of treatment in older patients with DLBCL or those with comorbidities with impressive results ${ }^{9}$. With the impending availability of safer and effective CART products, such as lisocaptagene maraleucel ${ }^{18}$ more patients may be referred for that therapy.

The greatest progress will result from the development of rational combinations with improved efficacy and a favorable safety profile. While the abovementioned trials are underway, identification of biomarkers are essential to help select individual patients for optimal therapies. Such novel chemo-free approaches will certainly lead to improved outcomes for patients with DLBCL and other histologies of B-cell lymphomas.

\section{Author details \\ 'Lymphoma Research Foundation, New York, NY, USA. 'Mayo Clinic Foundation, Rochester, MN, USA. ${ }^{3}$ Lymphoma Service, Memorial Sloan- Kettering Cancer Center, New York, NY, USA}

\section{Conflict of interest}

B.D.C.-Advisory boards/consulting-Morphosys, Karyopharm, BMS/Celgene, Kite, Epizyme, Beigene, Symbio, TG Therapeutics; G.N.—Celgene/BMS, Morphosys, Curis, Seattle Genetics, Kymera, Kite, Ryvu; G.S.-Morphosys, Abbvie, Autolus, Beigene, BMS/Celgene, Genmab, Roche, Janssen, Debiopharm, Milteniy, Novartis, Velosbio.

\section{Publisher's note}

Springer Nature remains neutral with regard to jurisdictional claims in published maps and institutional affiliations.

Received: 30 December 2020 Revised: 23 February 2021 Accepted: 3 March 2021

Published online: 05 April 2021

\section{References}

1. Armitage, J. O. \& Weisenburger, D. D. New approach to classifying nonHodgkin's lymphomas: clinical features of the major histologic subtypes. J. Clin. Oncol. 16, 2780-2795 (1998)

2. Feugier, P. et al. Long-term results of the R-CHOP study in the treatment of elderly patients with diffuse large B-cell lymphoma: a study by the Groupe d'Etude des Lymphomes de l'Adulte. J. Clin. Oncol. 23, 4117-4126 (2005).

3. Crump, M. et al. Outcomes in refractory diffuse large B-cell lymphoma: results from the international SCHOLAR-1 study. Blood. 130, 1800-1808 (2017).

4. Sehn, L. H. et al. Polatuzumab vedotin in relapsed or refractory diffuse large Bcell lymphoma. J. Clin. Oncol. 38, 155-165 (2020).

5. Kalakonda, N. et al. Selinexor in patients with relapsed or refractory diffuse large B-cell lymphoma (SADAL): a single-arm, multinational, multicentre, openlabel, phase 2 trial. Lancet Haematol. 7, e511-e522 (2020).

6. Salles, G. et al. Tafasitamab plus lenalidomide in relapsed or refractory diffuse large B-cell lymphoma (L-MIND): a multicentre, prospective, single-arm, phase 2 study. Lancet Oncol. 21, 978-988 (2020).

7. Locke, F. L. et al. Long-term safety and activity of axicabtagene ciloleucel in refractory large B-cell lymphoma (ZUMA-1): a single-arm, multicentre, phase 12 trial. Lancet Oncol. 20, 31-42 (2019).

8. Schuster, S. J. et al. Tisagenlecleucel in adult relapsed or refractory diffuse large B-cell lymphoma. N. Engl. J. Med. 380, 45-56 (2019).

9. Olszewski, A. J. et al. Single-agent mosunetuzumab is a promising safe and efficacious chemotherapy-free regimen for elderly/unfit patients with previously untreated diffuse large B-cell lymphoma. Blood. 136, abstr. 401 (2020).

10. Hutchings, M. et al. Glofitamab step-up dosing induces high response rates in patients with hard-to-treat refractory or relapsed non-Hodgkin lymphoma. Blood. 136, abstr. 403 (2020).

11. Hutchings, M. et al. Subcutaneous epcoritamab induces complete responses with an encouraging safety profile across relapsed/refractory B-cell nonHodgkin lymphoma subtypes, including patients with prior CAR-T therapy: updated dose escalationd data. Blood. 136, abstr. 402 (2020).

12. Bannerii, R. et al. Odronextamab (REGN1979), a human CD20 x CD3 bispecific antibody, induces durable, complete responses in patients with highly refractory B-cell non-Hodgkin lymphoma, including patients refractory to CAR T therapy. Blood. 136, abstr. 400 (2020).

13. Palanca-Wessels, M. C. et al. Safety and activity of the anti-CD79B antibodydrug conjugate polatuzumab vedotin in relapsed or refractory B-cell nonHodgkin lymphoma and chronic lymphocytic leukaemia: a phase 1 study. Lancet Oncol. 16, 704-715 (2015). 
14. Kuruvilla, J. et al. Selective inhibition of nuclear export with selinexor in patients with non-Hodgkin lymphoma. Blood. 129, 3175-3183 (2017).

15. Smith, S. D. \& Gopal, A. K. Selinexor for relapsed or refractory diffuse large B-cell lymphoma: examining the artifact. Lancet Haematol. 7, e707 (2020).

16. Engel, P. et al. Abnormal B lymphocyte development, activation, and differentiation in mice that lack or overexpress the CD19 signal transduction molecule. Immunity. 3, 39-50 (1995).

17. Kahl, B. S. et al. A phase I study of ADCT-402 (Loncastuximab Tesirine), a novel pyrrolobenzodiazepine-based antibody-drug conjugate, in relapsed/refractory B-cell non-Hodgkin Iymphoma. Clin. Cancer Res. 25 6986-6994 (2019)

18. Abramson, J. S. et al. Lisocabtagene maraleucel for patients with relapsed or refractory large B-cell lymphomas (TRANSCEND NHL 001): a multicentre seamless design study. Lancet. 396, 839-852 (2020).

19. Hamadani, M. et al. Final results of a phase 1 study of loncastuximab tesirine in relapsed/refractory B-cell non-Hodgkin lymphoma. Blood (2020). Online ahead of print.

20. Woyach, J. A. et al. A phase 1 trial of the Fc-engineered CD19 antibody XmAb5574 (MOR00208) demonstrates safety and preliminary efficacy in relapsed CLL. Blood. 124, 3553-3560 (2014)

21. Staber, P. B. et al. Primary analysis of anti-CD19 tafasitamab (MOR208) treatment in combination with idelalisib or venetoclax in R/R CLL patients who failed prior BTK inhibitor therapy (COSMOS Trial). Blood. 134, abstr. 1754 (2019).
22. Jurczak, W. et al. Phase lla study of the CD19 antibody MOR208 in patients with relapsed or refractory B-cell non-Hodgkin's lymphoma. Ann. Oncol. 29, 1266-1272 (2018)

23. Salles, G. et al. Long-term outcomes from the phase ॥ L-MIND study of tafasitamab (MOR208) plus lenalidomide in patients with relapsed or refractory diffuse large B-cell lymphoma. EHA Library. abstr. EP1201 (2020).

24. Cheson, B. D., Morschhauser, F. \& Martin, P. Management of adverse events from the combination of rituximab and lenalidomide in the treatment of patients with follicular and low-grade non-Hodgkin lymphoma. Clin. Lymphoma Myeloma Leuk. 20, 563-571 (2020).

25. Nowakowski, G. S. et al. RE-MIND study: a propensity score-based 1:1 matched comparison of tafasitamab + lenalidomide (L-MIND) versus lenalidomide monotherapy (real world data) in transplant-ineligible patients with relapsed/ refractory (R/R) diffuse large B-cell lymphoma (DLBCL). J. Clin. Oncol. 38, 8020 (2020).

26. Wang, $\mathrm{M}$. et al. Oral lenalidomide with rituximab in relapsed or refractory diffuse large cell, follicular and transformed lymphoma: a phase II clinical trial. Leukemia. 27, 1902-1909 (2013).

27. Zinzani, P. L. et al. Combination of lenalidomide and rituximab in elderly patients with relapsed or refractory diffuse large B-cell lymphoma: a phase 2 trial. Clin. Lymphoma Myeloma Leuk. 11, 462-466 (2011).

28. Neelapu, S. S. et al. Interim analysis of ZUMA-12: a phase 2 study of axicaptagene ciloleucel (Axi-Cel) as first-line therapy in patients (pts) with highrisk large B cell lymphoma (LBCL). Blood. 136, abstr. 405 (2020). 